\title{
Accurate Mapping of Multilevel Rydberg Atoms on Interacting Spin-1/2 Particles for the Quantum Simulation of Ising Models
}

\author{
Sylvain de Léséleuc, ${ }_{1}$ Sebastian Weber, ${ }^{2}$ Vincent Lienhard, ${ }^{1}$ Daniel Barredo, ${ }_{1}^{1}$ Hans Peter Büchler, ${ }^{2}$ \\ Thierry Lahaye, ${ }^{1}$ and Antoine Browaeys ${ }^{1}$ \\ ${ }^{1}$ Laboratoire Charles Fabry, Institut d'Optique Graduate School, CNRS, Université Paris-Saclay, 91127 Palaiseau Cedex, France \\ ${ }^{2}$ Institute for Theoretical Physics III and Center for Integrated Quantum Science and Technology, University of Stuttgart, \\ 70550 Stuttgart, Germany
}

(Received 17 October 2017; published 16 March 2018)

\begin{abstract}
We study a system of atoms that are laser driven to $n D_{3 / 2}$ Rydberg states and assess how accurately they can be mapped onto spin-1/2 particles for the quantum simulation of anisotropic Ising magnets. Using nonperturbative calculations of the pair potentials between two atoms in the presence of electric and magnetic fields, we emphasize the importance of a careful selection of experimental parameters in order to maintain the Rydberg blockade and avoid excitation of unwanted Rydberg states. We benchmark these theoretical observations against experiments using two atoms. Finally, we show that in these conditions, the experimental dynamics observed after a quench is in good agreement with numerical simulations of spin-1/2 Ising models in systems with up to 49 spins, for which numerical simulations become intractable.
\end{abstract}

DOI: 10.1103/PhysRevLett.120.113602

A promising approach for quantum information science and quantum simulation relies on single atoms trapped in optical tweezers and excited to Rydberg states [1]. Recent experimental progress has demonstrated active loading of up to 50 atoms in arrays of optical tweezers arranged in arbitrary geometries $[2,3]$. The strong interactions between Rydberg atoms (van der Waals or dipolar exchange) make these systems ideal for quantum simulation of spin Hamiltonians [1,4], lattice gauge theories [5], or topological matter $[6,7]$.

A major ingredient to realize such pristine artificial systems is the identification of suitable Rydberg levels and a full characterization of the interaction potentials. In the simplest case one identifies the ground state as the spindown state $|\downarrow\rangle$ and the Rydberg excitation as the spin-up state $|\uparrow\rangle$ for the implementation of spin-1/2 Hamiltonians [8-11]. However, in practice, describing the atom as a twolevel system is an approximation that can be difficult to fulfill due to small splittings between levels in the Rydberg manifold. For a single atom, it is sufficient to apply a magnetic field of a few Gauss to isolate a single two-level transition. But already for two atoms, the density of pair states becomes large, and, due to interactions, mixing between different levels occurs in configurations without special symmetries (Fig. 1). Finding optimal parameters such that the system is accurately described as a spin- $1 / 2$ system with a well-defined interaction potential is thus nontrivial and needs to be addressed in view of applications in quantum simulation.

A natural choice for implementing spin Hamiltonians with $\mathrm{Rb}$ atoms is to use $n S$ Rydberg states [11], as they possess only two Zeeman sublevels and do not feature Förster resonances [12]. However, many experiments use $n P$ or $n D$ states: the former are the only ones accessible from the ground state using single-photon dipole transitions $[13,14]$ and are used in particular for Rydberg dressing [15-18], while the latter $[10,19,20]$ require less laser power for excitation from the ground state as compared to $n S$ states. Moreover, for both $n P$ and $n D$ states, the van der Waals interaction can be anisotropic, opening the way for simulating exotic matter $[4,5]$. Nevertheless when implementing an anisotropic Ising model with $n D_{3 / 2}$ states, deviations from the prediction of a spin-1/2 model can occur, as we observed in Ref. [10].

In this Letter, we focus on Rydberg $n D_{3 / 2}$ states, and derive under which conditions the picture of a spin- $1 / 2$ model with an effective anisotropic interaction potential is valid, despite the large number of Rydberg levels involved. For that purpose, we use recent software [21,22] to calculate the exact pair-state potentials in the presence of external electric and magnetic fields. We find a remarkable sensitivity of the interaction spectrum to weak static electric fields, which can lead to a breakdown of the Rydberg blockade not considered in previous studies [12,23-27]. We then experimentally corroborate this prediction in a simple system of two atoms. Finally, we extend our study to a ring of 8 atoms and a $7 \times 7$ square array, where deviations from the spin-1/2 model were observed in Ref. [10], and now demonstrate a much better agreement with a numerical simulation of the spin- $1 / 2$ model. We believe that our results could stimulate similar studies in other quantum 
simulation platforms, such as ultracold molecules, trapped ions, or solid-state devices.

We use the Rydberg state $|r\rangle=\left|n D_{3 / 2}, m_{J}=3 / 2\right\rangle$ and couple it to the ground state $|g\rangle=\left|5 S_{1 / 2}, F=2, m_{F}=2\right\rangle$ by a two-photon transition [Fig. 1(b)]. Ideally we want to identify the states $|g\rangle$ and $|r\rangle$ with pseudo-spin-1/2 states $|\downarrow\rangle$ and $|\uparrow\rangle$. In this case, when taking into account interactions between atoms in $|r\rangle$, the system maps onto an Ising-like model in a transverse field [8-11] governed by the Hamiltonian

$$
H=\sum_{i} \frac{\hbar \Omega}{2} \sigma_{x}^{i}+\frac{1}{2} \sum_{i \neq j} U_{i j} n_{i} n_{j}
$$

Here, $\Omega$ is the Rabi frequency corresponding to the laser driving, $\sigma_{x}^{i}=|r\rangle\left\langle\left. g\right|_{i}+\mid g\right\rangle\left\langle\left. r\right|_{i}\right.$ and $\left.n_{i}=\mid r\right\rangle\left\langle\left. r\right|_{i}\right.$, and the rotating wave approximation has been applied. The interaction between atoms $i$ and $j$ is given at large distances by an anisotropic van der Waals potential $U_{i j}=C_{6}\left(\theta_{i j}\right) / R_{i j}^{6}$, where $R_{i j}$ is the interatomic distance and $\theta_{i j}$ the angle between the internuclear axis and the quantization axis, see Fig. 1(a).

We look for conditions allowing us to describe the interaction spectrum for a pair of atoms by a single potential curve $U(R, \theta)$ as in Fig 1(c). To approach this problem quantitatively, we diagonalize the dipole-dipole Hamiltonian [28] (and higher-order multipole contributions) in the presence of arbitrary external electric and magnetic fields [21]. In view of reproducing the experiment of Ref. [10], we chose the state $|r\rangle=\left|61 D_{3 / 2}, m_{J}=3 / 2\right\rangle$. Figure 2 shows the interaction spectrum for a generic angle $\theta=78^{\circ}$. The shading of the interaction potentials shows

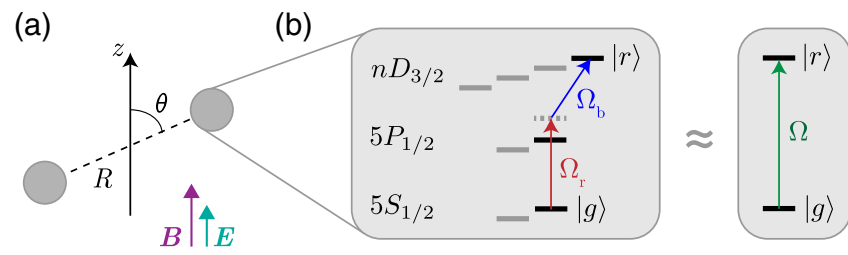

(c)

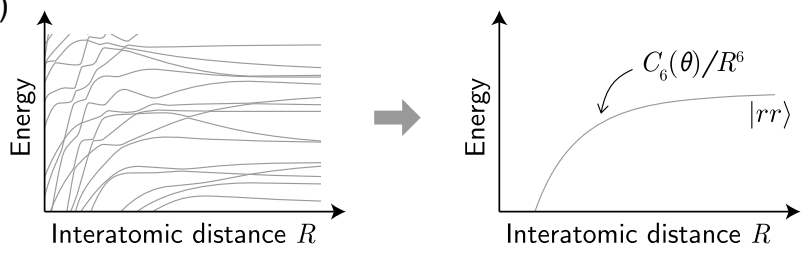

FIG. 1. Mapping a system of multilevel Rydberg atoms onto a spin-1/2 model. (a) System: Two atoms separated by a distance $R$; $\theta$ is the angle between the interatomic axis and the quantization axis $z$ defined by a magnetic field $B$. An electric field $E$ can be applied along $z$. (b) A two-photon transition couples coherently the ground state $|g\rangle$ to a target Rydberg state $|r\rangle$ with an effective two-photon Rabi frequency $\Omega$. (c) Full energy spectrum of the atom pair. The mapping consists in replacing this complex structure by an effective interaction potential. the overlap with $|r r\rangle$. In (a), no magnetic and electric fields are applied, and some Zeeman pair states interact weakly, while being still coupled to $|g g\rangle$. Consequently, the Rydberg blockade is broken as the double excitation of Rydberg states is possible even at short distances [23,24]. Panel (b) shows the potentials in the presence of a magnetic field $B=-6.9 \mathrm{G}$. The Zeeman effect splits the various potentials and the state $|r r\rangle$ is now isolated from the other eigenstates. However, since the sign of the Zeeman shift is identical to that of the van der Waals interaction, for specific values of the interatomic distance $R$ the laser excitation of other Zeeman pair states is resonant; these "magic distances" [26,29] can lead to a breakdown of the blockade. In order to avoid this, one can use an opposite value for $B$ [panel (c), where $B=6.9 \mathrm{G}$ ]. These parameters are similar to the ones used in Ref. [10], and in these conditions, it is a good approximation to describe the system by a single state for $R>6 \mu \mathrm{m}$.

It turns out, however, that the interaction potentials are extremely sensitive to electric fields $E$. Figure 2(d) corresponds to the same parameters as in (c), but in the presence of a field $E=20 \mathrm{mV} / \mathrm{cm}$ along $z$. A naive calculation of the Stark shift of pair states for this value of $E$ would give shifts in the $100 \mathrm{kHz}$ range, which would have hardly any
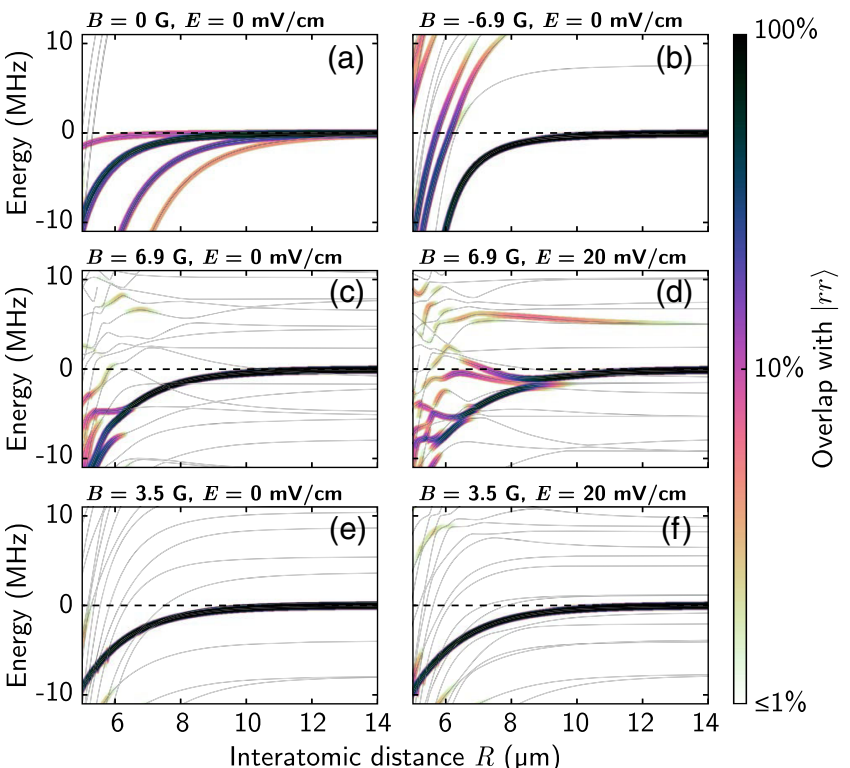

FIG. 2. Influence of magnetic and electric fields on the interaction potentials around the pair-state $|r r\rangle$ where $|r\rangle=\left|61 D_{3 / 2}, m_{j}=3 / 2\right\rangle$, for $\theta=78^{\circ}$. The shading encodes the overlap of the eigenstates with the noninteracting state $|r r\rangle$. (a) $B=0$ and $E=0:|r r\rangle$ overlaps with all the degenerate Zeeman pair states. (b) $B=-6.9 \mathrm{G}$ and $E=0$ : the interaction curves are split due to the Zeeman effect. Some curves still strongly mix with $|r r\rangle$ due to the interaction. (c) $B=6.9 \mathrm{G}$ and $E=0$ : one potential curve dominates. However, (d) the addition of a small electric field $E=20 \mathrm{mV} / \mathrm{cm}$ is enough to strongly perturb the pair states. (e),(f) This behavior is absent for $B=3.5 \mathrm{G}$. 
influence. However, exact diagonalization shows that the potentials are strongly affected, with many states being resonant with the excitation laser. We thus expect a significant breakdown of the Rydberg blockade in these conditions. Remarkably, this effect is absent for $B=3.5 \mathrm{G}$, see Figs. 2(e) and 2(f). In the optimal regime where a single potential curve $U(R, \theta)$ can be identified, we check if we can describe it by a van der Waals potential with an angular dependence $C_{6}(\theta) / R^{6}$. Figure 3(a) shows the energy dependence as a function of $R$ for $\theta=78^{\circ}$ together with a $1 / R^{6}$ fit. We observe, that for $R \gtrsim 8 \mu \mathrm{m}$, the van der Waals description is an excellent approximation. Figure 3(b) shows the angular dependence of $C_{6}(\theta)$. We have thus extended the anisotropic effective potential approach of Refs. [26,30] beyond the strong blockade regime.

We now turn to the experimental test of these predictions. Our setup has been described elsewhere [10]: we create two-dimensional arrays of optical tweezers loaded with single atoms from a magneto-optical trap (MOT). Active sorting of the atoms yields fully-loaded arrays [2] with up to 49 atoms. The electric field $E$ at the position of the atoms is controlled by a set of 8 electrodes under vacuum [31]; we zero out the electric field with an accuracy better than $|E|=5 \mathrm{mV} / \mathrm{cm}$ by performing Stark spectroscopy on high- $n$ Rydberg states [32]. We optically pump the atoms into $|g\rangle$ in the presence of a magnetic field pointing along the $z$ axis, within the arrays' plane. We then switch off the tweezers and apply a Rydberg excitation pulse of duration $\tau$ (we use a two-photon transition with lasers at 795 and $475 \mathrm{~nm}$ with an effective Rabi frequency $\Omega=2 \pi \times 1.2 \mathrm{MHz}$ ). At the end of the sequence, we switch on again the tweezers. Atoms in $|g\rangle$ are recaptured while those that have been excited to Rydberg states (either in $|r\rangle$ or in any other Rydberg state) are repelled by the traps and lost $[10,33]$. Thus, when we switch on again the MOT beams, atoms in $|g\rangle$ are observed by fluorescence, while missing atoms are assigned to Rydberg states.

As a first test of the influence of electric and magnetic fields on the potentials, we perform two-atom blockade experiments $[19,20]$ with $R=6.5 \mu \mathrm{m}$ and $\theta=78^{\circ}$, i.e., the same parameters as in Fig. 2. We use four different settings
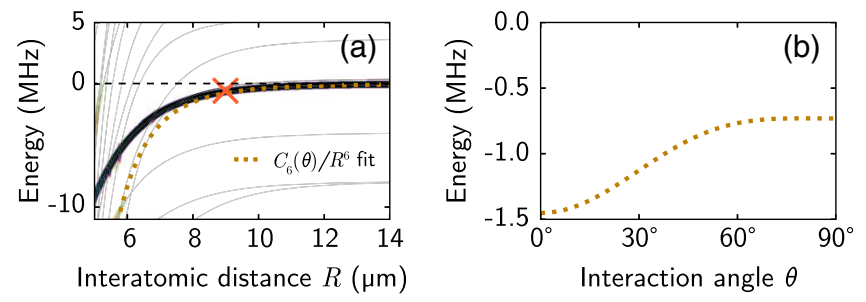

FIG. 3. Approximation of the interaction by an anisotropic van der Waals potential $C_{6}(\theta) / R^{6}$. (a) Comparison of the exact interaction energy (solid line) with the asymptotic determination of the van der Waals potential (dashed line) for a fixed angle $\theta=78^{\circ}$ and $B=3.5 \mathrm{G}$. (b) Angular dependence of $C_{6}(\theta) / R^{6}$ at $R=9 \mu$ m marked by the cross on (a). of the external fields: $B$ is either 3.5 or $6.9 \mathrm{G}$, and $E$ either zero (within the accuracy $\sim 5 \mathrm{mV} / \mathrm{cm}$ of our cancellation of stray fields) or $20 \mathrm{mV} / \mathrm{cm}$. In order to quantify the Rydberg blockade, we measure the probability $P_{r r}$ to have two Rydberg excitations (in $|r\rangle$ or in any other Rydberg level) after the excitation pulse. The results are displayed in Fig. 4. We observe, as expected, a strong suppression of $P_{r r}$ for all settings, except for $B=6.9 \mathrm{G}$ and $E=20 \mathrm{mV} / \mathrm{cm}$, where we find a significant probability to excite the two atoms. To compare with theory, we simulate the dynamics of the two-atom system solving the Schrödinger equation and calculate the probability to excite the two atoms [34]. We assume two different models to describe the interacting system: in the first one (Fig. 4 solid line), we use the full interaction spectrum and include around 800 pair-states within $2 \mathrm{GHz}$ from the resonance (a bigger electric field would drastically increase the basis size). In the second model (dashed line), we describe the interaction in the $|r r\rangle$ state with the single potential curve identified above, thus solving the spin-1/2 model governed by the Hamiltonian Eq. (1). This simulation with no adjustable parameter is in excellent agreement with the experimental data.

We now investigate more systematically how the geometry and the electric and magnetic fields affect the accuracy of the mapping on a spin-1/2 model. Using the exact simulation taking into account the full interaction spectrum, as done for Fig. 4, we calculate the average value of the double excitation probability $P_{r r}$ at long times and look at the range of parameters for which $P_{r r}$ remains small. Figure 5(a) corresponds to $E=0$, while we show in (b) a "worst-case scenario" where $E$ is chosen in the range
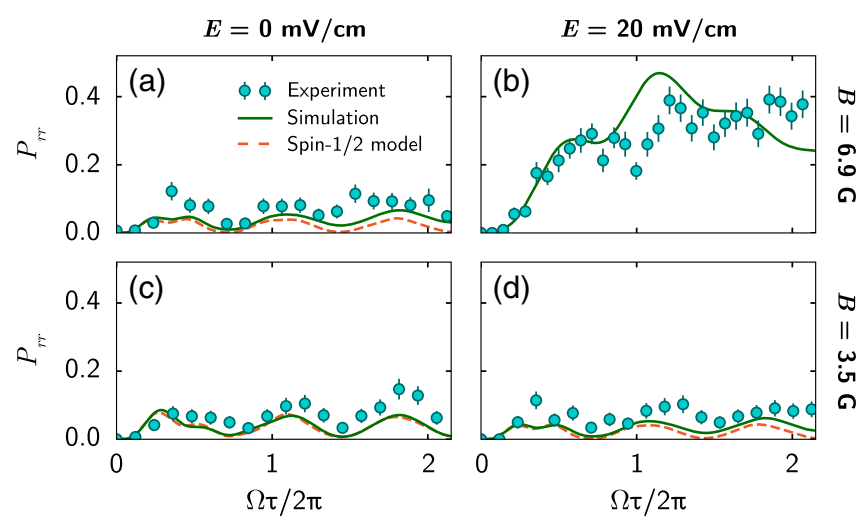

FIG. 4. Two-atom blockade experiments. Probability $P_{r r}$ to excite the two atoms as a function of the pulse area $\Omega \tau$. For $B=6.9 \mathrm{G}[(\mathrm{a}),(\mathrm{b})]$ increasing $E$ from 0 to $20 \mathrm{mV} / \mathrm{cm}$ breaks the Rydberg blockade. At $B=3.5 \mathrm{G}[(\mathrm{c}),(\mathrm{d})]$ an efficient blockade is maintained, even in the presence of the electric field. The solid lines result from a simulation taking into account the full interaction spectrum (see text). The dashed lines are obtained by modeling the atoms as spin- $1 / 2$ particles with a single interaction potential for $|r r\rangle$, except in case (b) where the pair state is too perturbed. The error bars show the standard error of the mean. 


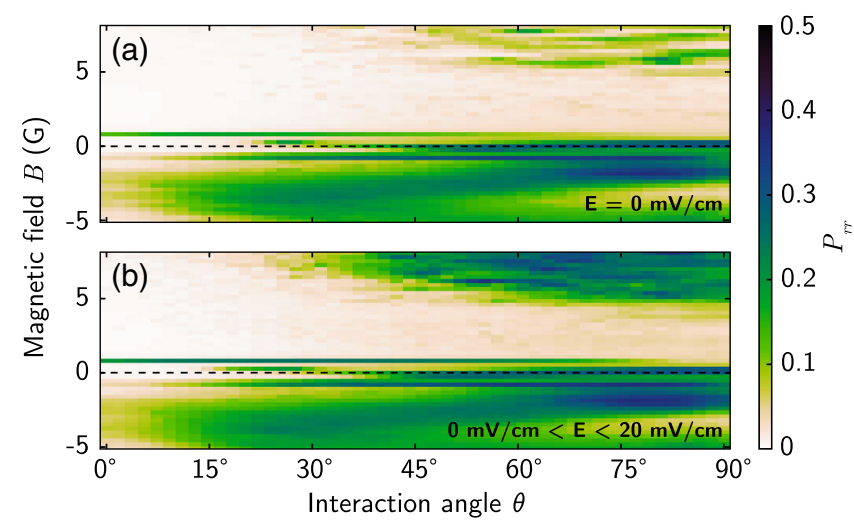

FIG. 5. Influence of $\theta, B, E$ on the mapping onto a spin-1/2 system. Calculated probability of double excitations at long times (see text) as a function of the magnetic field $B$ and the angle $\theta$. The interatomic distance is fixed at $R=6.5 \mu \mathrm{m}$. The electric field is $E=0$ in (a) and chosen between 0 and $20 \mathrm{mV} / \mathrm{cm}$ such that the probability for two Rydberg excitations is maximized in (b).

$0-20 \mathrm{mV} / \mathrm{cm}$ so as to maximize $P_{r r}$. For $\theta \approx 0$ the system is faithfully described by a spin- $1 / 2$ system. For increasing $\theta$, we identify the range of magnetic fields where Rydberg blockade is maintained. In addition, we observe a breaking of the Rydberg blockade for negative $B$ as predicted in
Ref. [26]. A similar analysis for various principal quantum numbers $n$ indicates that the presence of a Förster resonance at $n=59$ is responsible for this sensitivity to weak electric fields $[29,31]$.

Now that we have identified parameters allowing us to map our two-atom system onto a spin-1/2 model, we extend the study to larger systems. We first revisit the experimental realization of an 8-atom ring, reported in Ref. [10], where we observed a discrepancy with the spin$1 / 2$ model. We apply a Rydberg excitation pulse and observe the ensuing dynamics by measuring the fraction $f_{R}$ of atoms that are excited to Rydberg states. We also extract the probability $P_{5+}$ that more than five atoms are excited, i.e., that the blockade condition is violated, as, for our parameters, nearest-neighbor excitation is thwarted. Prior to this experiment we compensated the stray electric field better than $5 \mathrm{mV} / \mathrm{cm}$. Figures $6(\mathrm{a})-6$ (c) show the results for two values of the magnetic field. For $B=6.9 \mathrm{G}$, we observe a slow rise of $P_{5+}$ above the prediction of the spin$1 / 2$ model. Contrarily, for $B=3.5 \mathrm{G}$, we find a much better agreement with the spin-1/2 model as expected from above.

We then probe a square array of $7 \times 7$ atoms [Figs. 6(d)6(f)]. As an exact simulation of the dynamics of the 49-atom system is no longer possible, we use the fact that two neighboring atoms cannot be excited due to the (a)

(d)

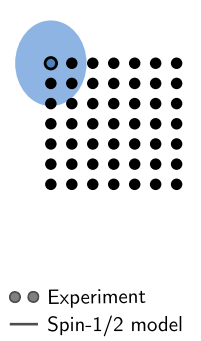

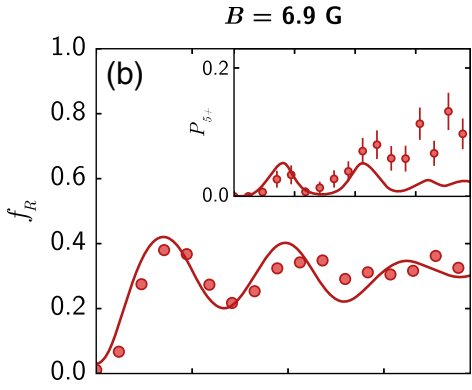

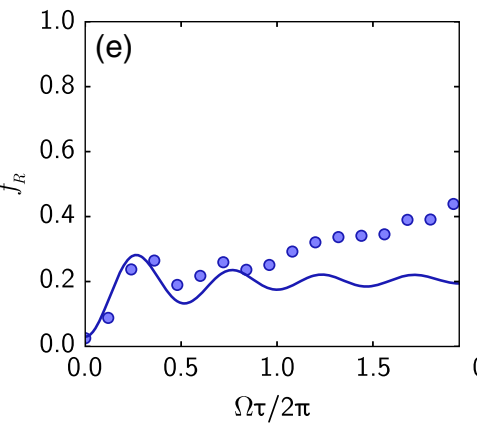

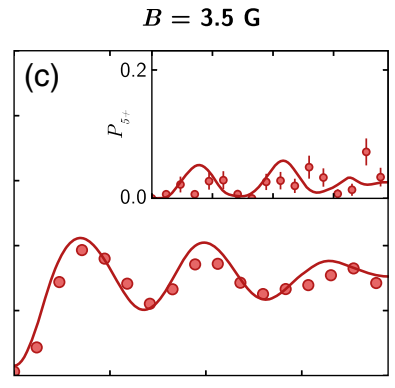

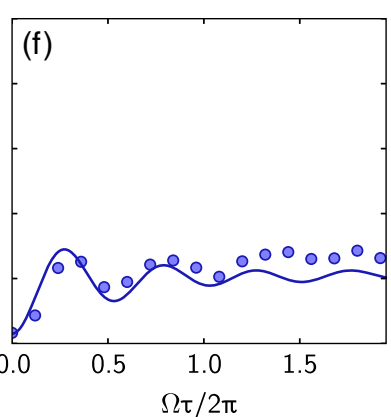

FIG. 6. Dynamics of an ensemble of atoms under Rydberg excitation. (a) 8-atom ring with a nearest neighbor spacing of $6.5 \mu$ m. The shaded ellipse illustrates the range of the anisotropic blockaded region $U>\hbar \Omega$. (b) Evolution of the Rydberg fraction $f_{R}$ with the pulse area $\Omega \tau$ for $B=6.9 \mathrm{G}$. The inset shows the probability $P_{5+}$ to observe configurations with at least 5 excitations. At large times, the experimental points systematically lie above the results of a simulation of the corresponding spin-1/2 model (solid line). (c) Same parameters with $B=3.5 \mathrm{G}$. (d) Square lattice of $7 \times 7$ traps (lattice spacing $6.1 \mu \mathrm{m}$ ). The blockade extends over nearest and nextnearest neighbors. (e) Evolution of the Rydberg fraction for $B=6.9 \mathrm{G}$. Here the data show a slow increase in $f_{R}$ at long times, while the spin-1/2 model predicts a saturation. (f) For $B=3.5 \mathrm{G}$, the agreement with the spin- $1 / 2$ model becomes very good. All figures: error bars depict the standard error of the mean and are often smaller than the symbol size. 
Rydberg blockade to truncate the Hilbert space from $2^{49}$ to $\sim 2^{30}$ states. We have checked with systems of up to 25 atoms that the truncation gives the same results as an exact calculation. We solve the time-dependent Schrödinger equation using a split-step approach. Again, we experimentally find a deviation with respect to the spin$1 / 2$ model for $B=6.9 \mathrm{G}$, while at lower $B$ the agreement is much better. We have thus identified the conditions where our system can be used as a quantum simulator of an anisotropic spin-1/2 Ising model.

In conclusion, we have explored the mapping on spin$1 / 2$ models of interacting multilevel Rydberg atoms by taking into account the details of the atomic structure in the presence of electric and magnetic fields. We searched for conditions under which the interaction between two Rydberg atoms can be faithfully described by a single potential curve. We found that this approximation can be sensitive to electric fields, thus extending previous studies on the breakdown of the blockade [12,23-27], and searched numerically for optimal parameters. Then, using atomic arrays of increasing size, from a pair of atoms to a $7 \times 7$ array, we confirmed that their dynamics is accurately reproduced by a spin-1/2 model with anisotropic Ising interaction. This work opens exciting prospects for harnessing the rich interaction spectrum of Rydberg atoms, for the engineering of various spin Hamiltonians-Ising, spinexchange, or $X X Z$ - as also proposed for polar molecules [35]. These insights could also help improve the control of interactions in Rydberg dressing experiments using $n P_{3 / 2}$ states [16], as well as for Rydberg slow light polaritons with $n D$ states [36].

This work benefited from financial support by the EU [H2020 FET-PROACT Project RySQ], by the PALM Labex (projects QUANTICA and XYLOS), and by the Région Île-de-France in the framework of DIM Nano-K.

S. d. L. and S. W. contributed equally to this work.

[1] M. Saffman, T. G. Walker, and K. Mølmer, Rev. Mod. Phys. 82, 2313 (2010).

[2] D. Barredo, S. de Léséleuc, V. Lienhard, T. Lahaye, and A. Browaeys, Science 354, 1021 (2016).

[3] M. Endres, H. Bernien, A. Keesling, H. Levine, E. R. Anschuetz, A. Krajenbrink, C. Senko, V. Vuletic, M. Greiner, and M. D. Lukin, Science 354, 1024 (2016).

[4] A. W. Glaetzle, M. Dalmonte, R. Nath, C. Gross, I. Bloch, and P. Zoller, Phys. Rev. Lett. 114, 173002 (2015).

[5] A. W. Glaetzle, M. Dalmonte, R. Nath, I. Rousochatzakis, R. Moessner, and P. Zoller, Phys. Rev. X 4, 041037 (2014).

[6] S. R. Manmana, E. M. Stoudenmire, K. R. A. Hazzard, A. M. Rey, and A. V. Gorshkov, Phys. Rev. B 87, 081106 (2013).

[7] D. Peter, N. Y. Yao, N. Lang, S. D. Huber, M. D. Lukin, and H. P. Büchler, Phys. Rev. A 91, 053617 (2015).
[8] P. Schauß, M. Cheneau, M. Endres, T. Fukuhara, S. Hild, A. Omran, T. Pohl, C. Gross, S. Kuhr, and I. Bloch, Nature (London) 491, 87 (2012).

[9] P. Schauß, J. Zeiher, T. Fukuhara, S. Hild, M. Cheneau, T. Macrì, T. Pohl, I. Bloch, and C. Gross, Science 347, 1455 (2015).

[10] H. Labuhn, D. Barredo, S. Ravets, S. de Léséleuc, T. Macrì, T. Lahaye, and A. Browaeys, Nature (London) 534, 667 (2016).

[11] H. Bernien, S. Schwartz, A. Keesling, H. Levine, A. Omran, H. Pichler, S. Choi, A. S. Zibrov, M. Endres, M. Greiner, V. Vuletić, and M. D. Lukin, Nature (London) 551, 579 (2017).

[12] A. Reinhard, T. C. Liebisch, B. Knuffman, and G. Raithel, Phys. Rev. A 75, 032712 (2007).

[13] D. Tong, S. M. Farooqi, J. Stanojevic, S. Krishnan, Y. P. Zhang, R. Côté, E. E. Eyler, and P. L. Gould, Phys. Rev. Lett. 93, 063001 (2004).

[14] A. M. Hankin, Y.-Y. Jau, L. P. Parazzoli, C. W. Chou, D. J. Armstrong, A. J. Landahl, and G. W. Biedermann, Phys. Rev. A 89, 033416 (2014).

[15] J. Zeiher, R. van Bijnen, P. Schauss, S. Hild, J.-Y. Choi, T. Pohl, I. Bloch, and C. Gross, Nat. Phys. 12, 1095 (2016).

[16] Y.-Y. Jau, A. M. Hankin, T. Keating, I. H. Deutsch, and G. W. Biedermann, Nat. Phys. 12, 71 (2016).

[17] J. Lee, M. J. Martin, Y. Y. Jau, T. Keating, I. H. Deutsch, and G. W. Biedermann, Phys. Rev. A 95, 041801 (2017).

[18] J. Zeiher, J.-Y. Choi, A. Rubio-Abadal, T. Pohl, R. van Bijnen, I. Bloch, and C. Gross, Phys. Rev. X 7, 041063 (2017).

[19] E. Urban, T. A. Johnson, T. Henage, L. Isenhower, D. D. Yavuz, T. G. Walker, and M. Saffman, Nat. Phys. 5, 110 (2009).

[20] A. Gaëtan, Y. Miroshnychenko, T. Wilk, A. Chotia, M. Viteau, D. Comparat, P. Pillet, A. Browaeys, and P. Grangier, Nat. Phys. 5, 115 (2009).

[21] S. Weber, C. Tresp, H. Menke, A. Urvoy, O. Firstenberg, H. P. Büchler, and S. Hofferberth, J. Phys. B 50, 133001 (2017).

[22] https://pairinteraction.github.io.

[23] T. G. Walker and M. Saffman, J. Phys. B 38, S309 (2005).

[24] T. G. Walker and M. Saffman, Phys. Rev. A 77, 032723 (2008).

[25] T. Pohl and P. R. Berman, Phys. Rev. Lett. 102, 013004 (2009).

[26] B. Vermersch, A. W. Glaetzle, and P. Zoller, Phys. Rev. A 91, 023411 (2015).

[27] A. Derevianko, P. Kómár, T. Topcu, R. M. Kroeze, and M. D. Lukin, Phys. Rev. A 92, 063419 (2015).

[28] N. Šibalić, J. Pritchard, C. Adams, and K. Weatherill, Comput. Phys. Commun. 220, 319 (2017).

[29] See Supplemental Material at http://link.aps.org/ supplemental/10.1103/PhysRevLett.120.113602 for details about the $n$-dependence of the mapping and about the $B$-dependence of pair-state spectroscopy.

[30] D. Barredo, S. Ravets, H. Labuhn, L. Béguin, A. Vernier, F. Nogrette, T. Lahaye, and A. Browaeys, Phys. Rev. Lett. 112, 183002 (2014).

[31] S. Ravets, H. Labuhn, D. Barredo, L. Beguin, T. Lahaye, and A. Browaeys, Nat. Phys. 10, 914 (2014). 
[32] The accuracy on the nulling of the $E$ field at a specific position is $0.5 \mathrm{mV} / \mathrm{cm}$. Over a region of $30 \times 30 \mu \mathrm{m}^{2}$, comparable to our system's size, we have measured that the $E$ field does not vary by more than $\pm 5 \mathrm{mV} / \mathrm{cm}$.

[33] S. de Léséleuc, D. Barredo, V. Lienhard, A. Browaeys, and T. Lahaye, arXiv:1802.10424.

[34] We include the residual shot-to-shot fluctuations of the interatomic distance by averaging the result of the
Schrödinger equation over 200 realizations of the random positions of the atoms in their tweezers.

[35] D. Peter, S. Müller, S. Wessel, and H. P. Büchler, Phys. Rev. Lett. 109, 025303 (2012).

[36] C. Tresp, P. Bienias, S. Weber, H. Gorniaczyk, I. Mirgorodskiy, H. P. Büchler, and S. Hofferberth, Phys. Rev. Lett. 115, 083602 (2015). 